\title{
Controllability of Continuous Bimodal Linear Systems
}

\author{
Josep Ferrer, Juan R. Pacha, and Marta Peña \\ Deptartament de Matemática Aplicada I, Universitat Politècnica de Catalunya, Diagonal 647, 08028 Barcelona, Spain
}

Correspondence should be addressed to Juan R. Pacha; juan.ramon.pacha@upc.edu

Received 25 January 2013; Revised 12 April 2013; Accepted 17 April 2013

Academic Editor: Rongni Yang

Copyright (C) 2013 Josep Ferrer et al. This is an open access article distributed under the Creative Commons Attribution License, which permits unrestricted use, distribution, and reproduction in any medium, provided the original work is properly cited.

\begin{abstract}
We consider bimodal linear systems consisting of two linear dynamics acting on each side of a given hyperplane, assuming continuity along the separating hyperplane. We prove that the study of controllability can be reduced to the unobservable case, and for these ones we obtain a simple explicit characterization of controllability for dimensions 2 and 3 , as well as some partial criteria for higher dimensions.
\end{abstract}

\section{Introduction}

A switched linear system (see, e.g., [1]) is a hybrid system (see, e.g., [2]) which consists of several linear subsystems and a rule that orchestrates the switching among them. The classical piecewise linear systems (see [3]) occur when the switching law depends only on the state. In recent years, piecewise linear dynamical/control systems have again attracted the attention of the researchers, indeed, because they are the most natural extension to linear systems in order to capture nonlinear phenomena as, for instance, limit cycles, heteroclinic and homoclinic orbits, and strange attractors. Also, their application has been especially successful in many engineering problems, such as the analysis and design of electronic oscillators (see [4]) or control systems.

They consist of two or more linear subsystems, each one acting in a different region-separated from the others by hypersurfaces-of the entire state space. In the basic case of bimodal systems, a couple of linear subsystems act at each side of a hyperplane. One says that the piecewise linear system is continuous if both adjacent subsystems coincide on the separating hypersurface.

For elementary circuits (see [4]), the number of state variables is typically two or three. Moreover, most of the nonlinear behavior appears already in low dimensions, even in the bimodal case. For example, the study in [5] gives a complete characterization of the focus-center limit cycle bifurcation for planar bimodal linear systems. Later works (see [6-8]) extended this analysis to the 3D case. Contrarily to what happens in $2 \mathrm{D}$, in $3 \mathrm{D}$, a remarkable phenomenon occurs, involving bimodal linear systems. Thus, whereas in $2 \mathrm{D}$, a point placed on the separating hyperplane is asymptotically stable when both subsystems are stable; in 3D, unstable global dynamics might arise under the same hypotheses.

Concerning structural stability (i.e., with regard to small perturbations), we point the reader to [9] for an account of generic properties and to [10] for a full characterization (both studies on the 2D case). About control systems see, for example, [11] (again for the planar case).

To investigate continuous bimodal systems, several authors have used reduced forms of the matrices involved, both for dynamical systems (e.g., in [4]) and control systems (e.g., in $[12,13])$. Here, we will use the reduced forms in [14, 15]. For a MAPLE program useful to compute them in $2 \mathrm{D}$ and 3D, see http://www.mal.upc.edu/joanr/html/cfbpwls.html.

The goal of this paper is to find explicit and effective criteria for controllability of continuous bimodal linear control systems (for a study of the discrete-time planar case, see [16], where explicit necessary and sufficient conditions for controllability and reachability are given). Other problems concerning discrete-time systems are considered in [17], where a controller is designed. In other cases as in [18] or in [19], the system is continuous and the controller is digital. A state feedback controller for piecewise linear systems is designed in [20]. Problems as stability and stabilization of switched systems are considered in $[18,21-23]$ or in [24]. In [25], the problem of controllability is addressed for linear time-invariant dynamical multiagent systems. Further contributions include switched systems with stochastic perturbations (see [26] and the references therein). In our 
approach, the key point in the study of the controllability relies on applying the implicit general conditions in [27] to the matrices in the reduced form given in [15]. We thoroughly achieve this objective for the 2D and 3D cases (Propositions 18 and 19) and obtain partial criteria for higher dimensions (Lemma 12 and Proposition 14).

We stress that we have included the unobservable case (which appears in a natural way, e.g., when considering parametrized families of systems), and we have proved that the study of the controllability for observable systems can be reduced to the unobservable ones (Proposition 17).

The structure of this paper is as follows. First, in Section 2, we recall the definition of continuous bimodal linear control system (CBLCS), the concept of controllability, and the implicit criterion in [27]. In Section 3, we provide the reduced forms for the matrices defining the subsystems of the CBLCS (see [15]). Later on, in Section 4 we obtain some partial criteria for controllability and uncontrollability of observable systems in higher dimensions and show that observable systems can, generically, become unobservable by adding the appropriate feedback (previously, Definitions 10 and 15 suit the necessary notions of observability and feedback system to the present context). As feedbacks preserve controllability (see Remark 16), there is no loss of generality in assuming that the system is unobservable. Subsequently, Section 5 tackles the controllability of unobservable CBLCS in dimensions 2 and 3. Last section illustrates the above results with an example of a 3D system (an occasional unobservable circuit). To complete the paper, conclusions appear at the end.

To summarize, the main contribution of this paper is twofold. First, we prove that one can reduce the study of the controllability of continuous bimodal linear control systems to the study of the controllability of the unobservable ones. Secondly, we provide effective and explicit criteria for controllability. Our main motivation is to give effective tools for the study of the controllability of systems of this kind (such as circuits; see Section 6). In comparison with the previous criteria in [27], the characterization of controllability given in this paper is simple and explicit, especially for dimensions 2 and 3. Up to our knowledge, there are no criteria for the study of the controllability of the systems at hand focused on unobservable systems. In addition, we discuss the practical use of the theoretic results presented by means of an example.

Throughout the paper, $\mathbb{R}$ will denote the set of real numbers; $M_{n \times m}(\mathbb{R})$ the set of matrices having $n$ rows, $m$ columns, and entries in $\mathbb{R} ; M^{\top} \in M_{m \times n}(\mathbb{R})$ the transpose matrix of $M \in M_{n \times m}(\mathbb{R})$. We will simply write $M_{n}(R)$ when $m=n ; G l_{n}(\mathbb{R})$ will be the group of nonsingular matrices in $M_{n}(\mathbb{R})$ and $I_{n}$ will be its identity element. Finally, we will denote by $e_{1}, \ldots, e_{n}$ the natural basis of the Euclidean space $\mathbb{R}^{n}$.

\section{Preliminaries}

We recall that a (single) linear control system is a system of type

$$
\dot{x}(t)=A x(t)+B u(t),
$$

$A \in M_{n}(\mathbb{R}), B \in M_{n \times 1}(\mathbb{R})$, and $C \in M_{1 \times n}(\mathbb{R})$, where $x(t)=\left(x_{1}(t), \ldots, x_{n}(t)\right)^{\top}, x_{i}=x_{i}(t), i=1, \ldots, n$ are the state variables, and $u(t)$ is the control function. In 1960, Kalman (see [28]) introduced the concept of controllability (see Definition 3) and showed that it is equivalent to the controllability matrix, defined as

$$
\mathscr{C}(A, B):=\left(\begin{array}{lllll}
B & A B & A^{2} B & \cdots & A^{n-1} B
\end{array}\right),
$$

having maximal rank. Therefore, the above system is controllable if and only if

$$
\operatorname{rank} \mathscr{C}(A, B)=n .
$$

The notion of controllability (Definition 1 ) is valid as well for bimodal systems and Theorem 4 below (see [27]) generalizes the characterization (3) in an implicit form.

In general, a bimodal linear control system consists of two subsystems

$$
\dot{x}(t)=A_{i} x(t)+B_{i} u(t), \quad i=1,2
$$

acting on disjoint semispaces. It is called continuous if both subsystems coincide in the common separatrix boundary of these semispaces, which, in particular, implies that $B_{1}=B_{2}$. Hence, one defines the following.

Definition 1. A continuous bimodal linear control system (CBLCS) is a system of the form

$$
\begin{gathered}
\dot{x}(t)= \begin{cases}A_{1} x(t)+B_{1} u(t), & \text { if } y(t) \leq 0 \\
A_{2} x(t)+B_{2} u(t), & \text { if } y(t)>0\end{cases} \\
y(t)=C x(t),
\end{gathered}
$$

where $A_{1}, A_{2} \in M_{n}(\mathbb{R}) ; B_{1}, B_{2} \in M_{n \times 1}(\mathbb{R}) ; C \in M_{1 \times n}(\mathbb{R})$, satisfying that both subsystems in (5a) coincide for $y(t)=0$, that is to say

$$
A_{1} x=A_{2} x, \quad \text { whenever } C x=0 .
$$

Remark 2. In several real applications, the systems often include state delay (for recent works on switched systems with state delay, see [23, 29-33]). However, this aspect is beyond the scope of this paper and possible extensions in this sense are contemplated as future work.

As we have pointed, the notion of controllability in [28] is also valid for these systems, and so they are controllable if they can be driven from a given initial state to a given final state by means of an appropriate control. More precisely, we have the following.

Definition 3. One says that system (5a) and (5b) is (completely) controllable if for any pair of states $\left(x_{0}, x_{f}\right)$ there exists a locally integrable input $u(t)$ such that the solution of $(5 a)$ and (5b) transfers the state $x(0)=x_{0}$ to the state $x_{f}$, that is, $x(T)=x_{f}$ for some $T>0$.

Obviously, a necessary condition is $B \neq 0$. The next theorem extends to CBLCS the characterization of controllability given in [28] for single control systems. 
Theorem 4 (Çamhbel et al., 2004; see [27]). Consider a CBLCS defined by (5a) and (5b) and the assumption (6). Let one denote bye $\in M_{n \times 1}(\mathbb{R})$ the matrix such that $C C=A_{2}-A_{1}$. Then, this system is controllable if and only if the following conditions hold.

(C1) The pair $\left(A_{1},[B \mid e]\right)$ is controllable.

(C2) Whenever there exist $\lambda \in \mathbb{R}, v \in \mathbb{R}^{n} \backslash\{0\}$, and $\mu_{1}, \mu_{2} \in$ $\mathbb{R}^{2}$ such that

$$
\left(\begin{array}{ll}
v^{\top} & \mu_{i}
\end{array}\right)\left(\begin{array}{cc}
\lambda I_{n}-A_{i} & B \\
C & 0
\end{array}\right)=0, \quad i=1,2
$$

then, necessarily, $\mu_{1} \mu_{2}>0$.

Remark 5. Concerning the above theorem, two points are worth mentioning.

(1) Because of (6), $A_{2}-A_{1}$ is at most of rank 1 and the kernel of $C$ is contained in the kernel of $A_{2}-A_{1}$, and so the columns of $A_{2}^{\top}-A_{1}^{\top}$ belong to the span of $C^{\top} \epsilon$ $\mathbb{R}^{n}$. Therefore, there exists a matrix $e \in M_{n \times 1}(\mathbb{R})$ such that $e C=A_{2}-A_{1}$ (see [27]).

(2) In condition $(\mathrm{C} 1), \widehat{B}:=[B \mid e]$ denotes the $n \times 2$ matrix formed by the column matrices $B$ and $e$. Thus, this condition means that the controllability matrix $\mathscr{C}(A, \widehat{B})$ - see (2)-has full rank.

Our aim is to derive, from Theorem 4, explicit criteria for controllability by means of simplifying the involved matrices. Firstly, note that, as an immediate consequence of Definition 3, linear transformations of the state variables do not change the controllable character of the system. Hence, one can assume

$$
C=\left(\begin{array}{llll}
1 & 0 & \cdots & 0
\end{array}\right)
$$

so that the separating subspace is

$$
\operatorname{Ker} C=\left\{x \in \mathbb{R}^{n}: x_{1}=0\right\}
$$

and (6) in Definition 1 becomes

$$
A_{2} e_{i}=A_{1} e_{i}, \quad 2 \leq i \leq n
$$

namely, $A_{1}$ and $A_{2}$ differ only in their first column, $A_{1} e_{1}$ and $A_{2} e_{1}$, respectively, and

$$
\begin{aligned}
& e=A_{2} e_{1}-A_{1} e_{1} \\
& \operatorname{rank}\left(\left[S^{-1} B \mid S^{-1} e\right] S^{-1} A_{1} S\left[S^{-1} B \mid S^{-1} e\right] \cdots\left(S^{-1} A_{1} S\right)^{n-1}\left[S^{-1} B \mid S^{-1} e\right]\right) \\
& =\operatorname{rank} S^{-1}\left([B \mid e] \quad A_{1}[B \mid e] \cdots A_{1}^{n-1}[B \mid e]\right)=\operatorname{rank}\left([B \mid e] \quad A_{1}[B \mid e] \cdots A_{1}^{n-1}[B \mid e]\right) \text {. }
\end{aligned}
$$

From now on, these simplifications are assumed. Then we will refer to a CBLCS simply as the triple $\left(A_{1}, A_{2}, B\right)$ verifying (10).

\section{Admissible Basis Changes}

Here we introduce, in Definition 6, the admissible basis changes as those changes in the state variables space preserving the hyperplanes $x_{1}=k$ and hence the matrix $C=\left(\begin{array}{llll}1 & 0 & \cdots & 0\end{array}\right)$. Next, in Proposition 8, we see that Conditions (C1) and (C2) of Theorem 4 are invariant under these changes, so one can check the controllability on any set of transformed matrices. Finally, Theorem 11 introduces the specific reduced forms which we will use in the sequel.

Definition 6. Admissible basis changes are basis changes in the state space variables preserving the hyperplane $x_{1}=k$. One will write $\mathcal{S}$ for the subgroup of the corresponding matrices in $G l_{n}(\mathbb{R})$. Therefore,

$$
\begin{array}{r}
S \in \mathcal{S} S=\left(\begin{array}{cc}
1 & 0 \\
U & T
\end{array}\right), \quad T \in G l_{n-1}(\mathbb{R}), \\
U \in M_{n-1 \times 1}(\mathbb{R}) .
\end{array}
$$

Remark 7. We emphasize two points.

(1) The basis changes in Definition 6 transform the triple of matrices $\left(A_{1}, A_{2}, B\right)$ into $\left(A_{1}^{\prime}, A_{2}^{\prime}, B^{\prime}\right)=$ $\left(S^{-1} A_{1} S, S^{-1} A_{2} S, S^{-1} B\right)$, whereas $C^{\prime}=C S=C$.

(2) Moreover, these transformations are just the same as the ones in [15] for dynamical systems, so the reduced forms are also valid for CBLCS here.

Proposition 8. For a given CBLCS, admissible basis changes preserve Condition (C1) and the values $\mu_{1}, \mu_{2}$ in Condition (C2).

Proof. Consider a CBLCS defined by $\left(A_{1}, A_{2}, B\right)$. Let us see that the vector $e^{\prime}$ verifying $e^{\prime} C=A_{2}^{\prime}-A_{1}^{\prime}$ is just $e^{\prime}=S^{-1} e$ as follows:

$$
\begin{aligned}
\left(S^{-1} e\right) C & =\left(S^{-1} e\right)(C S)=S^{-1}\left(A_{2}-A_{1}\right) S \\
& =S^{-1} A_{2} S-S^{-1} A_{1} S=A_{2}^{\prime}-A_{1}^{\prime} .
\end{aligned}
$$

Then, the pair $\left(A_{1},[B \mid e]\right)$ is controllable if and only if
This proves that Condition $(\mathrm{Cl})$ is invariant under admissible basis changes. Besides, from

$$
\left(\begin{array}{cc}
\lambda I_{n}-S^{-1} A_{i} S & S^{-1} B \\
C S & 0
\end{array}\right)=\left(\begin{array}{cc}
S^{-1} & \\
& I_{n}
\end{array}\right)\left(\begin{array}{cc}
\lambda I_{n}-A_{i} & B \\
C & 0
\end{array}\right)\left(\begin{array}{cc}
S & \\
& I_{n}
\end{array}\right)
$$


it is clear that

$$
\left(\begin{array}{ll}
v^{t} & \mu_{i}
\end{array}\right)\left(\begin{array}{cc}
\lambda I_{n}-S^{-1} A_{i} S & S^{-1} B \\
C S & 0
\end{array}\right)=0
$$

is equivalent to

$$
\left(\begin{array}{ll}
v^{t} S^{-1} & \mu_{i}
\end{array}\right)\left(\begin{array}{cc}
\lambda I_{n}-A_{i} & B \\
C & 0
\end{array}\right)=0,
$$

and, hence, admissible basis changes also preserve the values of $\mu_{1}, \mu_{2}$ in Condition (C2).

From Proposition 8, it follows that one can check the controllability conditions of Theorem 4 on any triple of matrices $\left(A_{1}^{\prime}, A_{2}^{\prime}, B^{\prime}\right)$ obtained, via an admissible basis change, from the triple $\left(A_{1}, A_{2}, B\right)$ defining the CBLCS. Hence, there is no loss of generality in assuming that the matrices in $\left(A_{1}, A_{2}, B\right)$ are in the reduced form derived in [15] (see Remark 7 above). We recall this result for the reader's convenience.

Lemma 9 (Ferrer et al. 2010, see [15]). Given a CBLCS $\left(A_{1}, A_{2}, B\right)$, then

$$
\operatorname{Ker} \mathscr{L}_{1}=\operatorname{Ker} \mathscr{L}_{2} \subset \operatorname{Ker} C \text {, }
$$

where $\mathscr{L}_{1}, \mathscr{L}_{2}$ are the observability matrices, that is,

$$
\mathscr{L}_{i}:=\left(\begin{array}{c}
C \\
C A_{i} \\
\vdots \\
C A_{i}^{n-1}
\end{array}\right), \quad i=1,2 .
$$

Definition 10. Under the above conditions, one writes

$$
K:=\operatorname{Ker} \mathscr{L}_{1}=\operatorname{Ker} \mathscr{L}_{2} \subseteq \operatorname{Ker} C,
$$

and one defines

$$
r:=\operatorname{rank} \mathscr{L}_{1}=\operatorname{rank} \mathscr{L}_{2}=n-\operatorname{dim} K
$$

as the observability rank of the system. In particular, the system is observable if $r=n$.

Theorem 11 (Ferrer et al., 2010; see [15]). With the notation of Lemma 9 and Definition 10 above, one has the following.

(a) The subspace $K$ is invariant under the endomorphisms $\mathscr{A}_{1}$ and $\mathscr{A}_{2}$ corresponding to the matrices $A_{1}$ and $A_{2}$, and their restrictions coincide, that is,

$$
\left.\mathscr{A}_{1}\right|_{K}=\left.\mathscr{A}_{2}\right|_{K}
$$

and one will write J for the Jordan normal form of these restricted endomorphisms.

(b) There exists an admissible basis change such that the following matrices are obtained:

$$
A_{1}^{\prime}=S^{-1} A_{1} S=\left(\begin{array}{cc}
K_{1} & 0 \\
\widetilde{A}_{1} & J
\end{array}\right), \quad A_{2}^{\prime}=S^{-1} A_{2} S=\left(\begin{array}{ll}
K_{2} & 0 \\
\widetilde{A}_{2} & J
\end{array}\right),
$$

where the matrices $K_{1}, K_{2}, \widetilde{A}_{1}$, and $\widetilde{A}_{2}$ are given, respectively, by

$$
\begin{aligned}
K_{1}= & \left(\begin{array}{ccccc}
a_{1} & 1 & 0 & \cdots & 0 \\
a_{2} & 0 & 1 & \cdots & 0 \\
\vdots & \vdots & \vdots & \ddots & \vdots \\
a_{r-1} & 0 & 0 & \cdots & 1 \\
a_{r} & 0 & 0 & \cdots & 0
\end{array}\right), \\
K_{2}= & \left(\begin{array}{ccccc}
\bar{a}_{1} & 1 & 0 & \cdots & 0 \\
\bar{a}_{2} & 0 & 1 & \cdots & 0 \\
\vdots & \vdots & \vdots & \ddots & \vdots \\
\bar{a}_{r-1} & 0 & 0 & \cdots & 1 \\
\bar{a}_{r} & 0 & 0 & \cdots & 0
\end{array}\right), \\
\widetilde{A}_{1} & =\left(\begin{array}{ccccc}
a_{r+1}^{\prime} & 0 & 0 & \cdots & 0 \\
\vdots & \vdots & \vdots & \ddots & \vdots \\
a_{n}^{\prime} & 0 & 0 & \cdots & 0
\end{array}\right), \\
\widetilde{A}_{2} & =\left(\begin{array}{ccccc}
\bar{a}_{r+1}^{\prime} & 0 & 0 & \cdots & 0 \\
\vdots & \vdots & \vdots & \ddots & \vdots \\
\bar{a}_{n}^{\prime} & 0 & 0 & \cdots & 0
\end{array}\right),
\end{aligned}
$$

and hence the following hold.

(b1) The coefficients $a_{1}, \ldots, a_{r}, \bar{a}_{1}, \ldots, \bar{a}_{r}$ are univocally determined by imposing the following relations:

$$
Q_{A_{1}}(s)=Q_{K_{1}}(s) Q_{J}(s), \quad Q_{A_{2}}(s)=Q_{K_{2}}(s) Q_{J}(s),
$$

on the characteristic polynomials of $A_{1}, K_{1}, A_{2}$, $K_{2}$, and $J$.

(b2) The coefficients $a_{r+1}^{\prime}, \ldots, a_{n}^{\prime}, \bar{a}_{r+1}^{\prime}, \ldots, \bar{a}_{n}^{\prime}$ depend on the admissible basis change applied.

Moreover,

$$
\left(S^{-1} B\right)^{\top}=\left(\begin{array}{llll}
b_{1} & b_{2}^{\prime} & \cdots & b_{n}^{\prime}
\end{array}\right),
$$

where $b_{1}=C B$ and $b_{2}^{\prime}, \ldots, b_{n}^{\prime}$ also depend on the admissible basis change. In particular, $b_{1}=b_{2}^{\prime}=\cdots=$ $b_{n}^{\prime}=0$ if and only if $B=0$.

\section{General Explicit Criteria}

Firstly, from the results in Section 3, we derive in Lemma 12 and Proposition 13 general sufficient conditions for uncontrollability of CBLCS. Next, from Theorems 4 and 11, Proposition 14 provides a sufficient condition for controllability of observable systems. Finally, Proposition 17 states that, if an observable CBLCS does not satisfy the hypothesis of Proposition 14, one can bring it into an unobservable feedback system (see Definition 15) in order to study its controllability. 
Lemma 12. Let $\left(A_{1}, A_{2}, B\right)$ be a CBLCS and $K$ as in Theorem 11. If $B \in K$, then the system is uncontrollable. In particular, it is uncontrollable whenever $B=0$.

Proof. If $B \in K$, a trajectory having initial conditions in $K$ remains in $K$ whatever the applied control, and so the system is uncontrollable.

The next proposition states a more specific condition for uncontrollability. We recall that $r$ is the observability rank (see (21)).

Proposition 13. Let $\left(A_{1}, A_{2}, B\right)$ be a CBLCS in the form of Theorem 11; then, if $b_{1}=\cdots=b_{r}=0$, the system is uncontrollable.

Proof. The assertion is obvious for $r=n$. If $r<n$, it is clear that the last $n-r$ vectors of the admissible basis considered in Theorem 11 span the subspace $K$, and so the result follows from Lemma 12.

Now, we focus on observable systems.

Proposition 14. Let $\left(A_{1}, A_{2}, B\right)$ be an observable CBLCS in the form of Theorem 11. Then, if $b_{1}=\cdots=b_{n-1}=0, b_{n} \neq 0$, the system is controllable.

Proof. For observable CBLCS, $r=n$; thus, the reduced matrices in Theorem 11 take the following form:

$$
\begin{gathered}
A_{1}=\left(\begin{array}{ccccc}
a_{1} & 1 & 0 & \cdots & 0 \\
a_{2} & 0 & 1 & \cdots & 0 \\
\vdots & \vdots & \vdots & \ddots & \vdots \\
a_{n-1} & 0 & 0 & \cdots & 1 \\
a_{n} & 0 & 0 & \cdots & 0
\end{array}\right), \\
A_{1}=\left(\begin{array}{ccccc}
\bar{a}_{1} & 1 & 0 & \cdots & 0 \\
\bar{a}_{2} & 0 & 1 & \cdots & 0 \\
\vdots & \vdots & \vdots & \ddots & \vdots \\
\bar{a}_{n-1} & 0 & 0 & \cdots & 1 \\
\bar{a}_{n} & 0 & 0 & \cdots & 0
\end{array}\right), \quad B=\left(\begin{array}{c}
b_{1} \\
b_{2} \\
\vdots \\
b_{n-1} \\
b_{n}
\end{array}\right),
\end{gathered}
$$

from which it follows at once that $\operatorname{det}\left(\begin{array}{lll}B & A_{1} B & \ldots\end{array}\right.$ $\left.A_{1}^{n-1} B\right) \neq 0$, so Condition (C1) of Theorem 4 holds. Condition (C2) is also trivial, because no $v \neq 0$ makes the last $n$ columns of

$$
\left(\begin{array}{ll}
v^{\top} & \mu_{i}
\end{array}\right)\left(\begin{array}{cc}
\lambda I_{n}-A_{i} & B \\
C & 0
\end{array}\right): \lambda v_{2}-v_{1}, \ldots, \lambda v_{n}-v_{n-1}, v_{n} b_{n}
$$

equal 0 .

If the hypothesis in Proposition 14 fails, we can study the controllability of a given observable CBLCS on the corresponding unobservable one obtained by the feedback described in Proposition 17.

As in the classical case of (single) control systems, feedback transformations result from applying additional controls of the form $F x(t)$.
Definition 15. If $\left(A_{1}, A_{2}, B\right)$ is a CBLCS, one says that the system $\left(A_{1}+B F, A_{2}+B F, B\right)$ is the feedback system obtained from $\left(A_{1}, A_{2}, B\right)$ by means of the feedback $F \in M_{1 \times n}(\mathbb{R})$.

Remark 16. Obviously, feedback transformations preserve controllability.

Proposition 17. Let $\left(A_{1}, A_{2}, B\right)$ be an observable CBLCS which, again, one assumes to be in the form of Theorem 11 . If $b_{1}=\cdots=b_{j-1}=0, b_{j} \neq 0,1 \leq j<n$; the feedback system determined by the triple

$$
\left(A_{1}+B F, A_{2}+B F, B\right)
$$

with

$$
F=\left(\begin{array}{lllllll}
0 & \cdots & 0 & f_{j+1} & 0 & \cdots & 0
\end{array}\right), \quad f_{j+1}=-\frac{1}{b_{j}}
$$

is unobservable, having rank $r=j$ (recall Definition 15).

Proof. From the reduced forms of Theorem 11, it is clear that

$$
A_{1}+B F=\left(\begin{array}{ccccc|ccccc}
a_{1} & 1 & 0 & \cdots & 0 & 0 & 0 & 0 & \cdots & 0 \\
a_{2} & 0 & 1 & \cdots & 0 & 0 & 0 & 0 & \cdots & 0 \\
\vdots & \vdots & \vdots & \ddots & \vdots & \vdots & \vdots & \vdots & \ddots & \vdots \\
a_{j-1} & 0 & 0 & \cdots & 1 & 0 & 0 & 0 & \cdots & 0 \\
a_{j} & 0 & 0 & \cdots & 0 & 0 & 0 & 0 & \cdots & 0 \\
\hline a_{j+1} & 0 & 0 & \cdots & 0 & \star & 1 & 0 & \cdots & 0 \\
a_{j+2} & 0 & 0 & \cdots & 0 & \star & 0 & 1 & \cdots & 0 \\
\vdots & \vdots & \vdots & \ddots & \vdots & \vdots & \vdots & \vdots & \ddots & \vdots \\
a_{n-1} & 0 & 0 & \cdots & 0 & \star & 0 & 0 & \cdots & 1 \\
a_{n} & 0 & 0 & \cdots & 0 & \star & 0 & 0 & \cdots & 0
\end{array}\right),
$$

where the upper and lower left blocks are of sizes $j \times j$ and $(n-j) \times j$, respectively, and, hence,

$$
\begin{aligned}
r & =\operatorname{rank}\left(\begin{array}{c}
C \\
C\left(A_{1}+B F\right) \\
\vdots \\
C\left(A_{1}+B F\right)^{n-1}
\end{array}\right) \\
& =\operatorname{rank}\left(\begin{array}{cccc|ccc}
1 & 0 & \cdots & 0 & 0 & \cdots & 0 \\
\star & 1 & \cdots & 0 & 0 & \cdots & 0 \\
\vdots & \vdots & \ddots & \vdots & \vdots & \ddots & \vdots \\
\star & \star & \cdots & 1 & 0 & \cdots & 0 \\
\hline \star & \star & \cdots & \star & 0 & \cdots & 0 \\
\star & \star & \cdots & \star & 0 & \cdots & 0 \\
\vdots & \vdots & \ddots & \vdots & \vdots & \ddots & \vdots \\
\star & \star & \cdots & \star & 0 & \cdots & 0
\end{array}\right)=j,
\end{aligned}
$$

which closes the proof.

Taking into account this proposition, one concludes that, beyond the cases covered by Propositions 13 and 14, the study of the controllability can be restricted to the study of unobservable systems. 


\section{Explicit Criteria for $n=2$ and $n=3$}

With the above tools, we will obtain explicit simple criteria when $n=2$ (in Proposition 18) and $n=3$ (in Proposition 19).

Firstly, let us see that the criterion in [21] for observable planar systems is also valid for the unobservable ones.

Proposition 18. Let one consider a planar CBLCS defined by $\left(A_{1}, A_{2}, B\right)$, with

$$
A_{1}=\left(\begin{array}{ll}
a_{1} & a_{3} \\
a_{2} & a_{4}
\end{array}\right), \quad A_{2}=\left(\begin{array}{ll}
\bar{a}_{1} & a_{3} \\
\bar{a}_{2} & a_{4}
\end{array}\right), \quad B=\left(\begin{array}{l}
b_{1} \\
b_{2}
\end{array}\right) .
$$

One writes $\mathscr{C}_{1}, \mathscr{C}_{2}$ for the controllability matrices of the corresponding subsystems, that is,

$$
\mathscr{C}_{1}=\left(\begin{array}{ll}
B & A_{1} B
\end{array}\right), \quad \mathscr{C}_{2}=\left(\begin{array}{ll}
B & A_{2} B
\end{array}\right) .
$$

It turns out that

(i) the system is unobservable if and only if $a_{3}=0$;

(ii) then, it is controllable if and only if

$$
\operatorname{det} \mathscr{C}_{1} \operatorname{det} \mathscr{C}_{2}>0
$$

or, equivalently,

$$
b_{1} \neq 0, \quad\left(b_{1} a_{2}-a_{1} b_{2}+a_{4} b_{2}\right)\left(b_{1} \bar{a}_{2}-\bar{a}_{1} b_{2}+a_{4} b_{2}\right)>0 \text {. }
$$

Proof. (i) One checks immediately that $r=2$ if and only if $a_{3} \neq 0$ (see Definition 10). (ii) Take $a_{3}=0$. If $b_{1}=0$, the system is uncontrollable from Proposition 13. Now, assume $b_{1} \neq 0$. One can rewrite Condition (C2) of Theorem 4 in the following form:

$$
\begin{gathered}
b_{1} v_{1}+b_{2} v_{2}=0, \quad v_{2}\left(\lambda-a_{4}\right)=0, \\
v_{1}\left(\lambda-a_{1}\right)-v_{2} a_{2}+\mu_{1}=0 \quad \text { for } i=1, \\
b_{1} v_{1}+b_{2} v_{2}=0, \quad v_{2}\left(\lambda-a_{4}\right)=0, \\
v_{1}\left(\lambda-\bar{a}_{1}\right)-v_{2} \bar{a}_{2}+\mu_{2}=0 \quad \text { for } i=2,
\end{gathered}
$$

$\lambda \in \mathbb{R}$, and $\left(v_{1}, v_{2}\right) \neq(0,0)$ implies $\mu_{1} \mu_{2}>0$. Then,

$$
\begin{gathered}
v_{1}=b_{2}, \quad v_{2}=-b_{1}, \quad \lambda=a_{4}, \\
\mu_{1}=b_{2}\left(a_{4}-a_{1}\right)+b_{1} a_{2}, \quad \mu_{2}=b_{2}\left(a_{4}-\bar{a}_{1}\right)+b_{1} \bar{a}_{2} ;
\end{gathered}
$$

therefore, Condition (C2) of Theorem 4 is equivalent to (36). In addition, (36) implies Condition (C1) in the same theorem, because one can rewrite this condition as

$$
\begin{aligned}
& \operatorname{rank}\left(\begin{array}{cccc}
b_{1} & a_{1} b_{1} & \bar{a}_{1}-a_{1} & a_{1}\left(\bar{a}_{1}-a_{1}\right) \\
b_{2} & a_{2} b_{1}+a_{4} b_{2} & \bar{a}_{2}-a_{2} & a_{2}\left(\bar{a}_{1}-a_{1}\right)+a_{4}\left(\bar{a}_{2}-a_{2}\right)
\end{array}\right) \\
& \quad=2,
\end{aligned}
$$

which completes the proof of Proposition 18.

Finally, the proposition below details the case $n=3$.
Proposition 19. For $n=3$, let one consider an unobservable CBLCS defined by $\left(A_{1}, A_{2}, B\right)$, which one assumes to have the reduced form in Theorem 11 . Let one denote by $\mathscr{C}_{1}, \mathscr{C}_{2}$ the controllability matrices of both subsystems, that is,

$$
\mathscr{C}_{1}=\left(\begin{array}{lll}
B & A_{1} B & A_{1}^{2} B
\end{array}\right), \quad \mathscr{C}_{2}=\left(\begin{array}{lll}
B & A_{2} B & A_{2}^{2} B
\end{array}\right)
$$

and by $\operatorname{det}_{12}^{j k} \mathscr{C}_{i}, i=1,2$, the determinant of the submatrix corresponding to the rows $j, k$ and the two first columns.

(1) If $r=2$, the reduced forms are

$$
\begin{gathered}
A_{1}=\left(\begin{array}{ccc}
a_{1} & 1 & 0 \\
a_{2} & 0 & 0 \\
a_{3} & 0 & \lambda_{0}
\end{array}\right), \quad A_{2}=\left(\begin{array}{ccc}
\bar{a}_{1} & 1 & 0 \\
\bar{a}_{2} & 0 & 0 \\
\bar{a}_{3} & 0 & \lambda_{0}
\end{array}\right), \\
B=\left(\begin{array}{l}
b_{1} \\
b_{2} \\
b_{3}
\end{array}\right),
\end{gathered}
$$

and then one has the following.

(1.1) If $b_{1}=b_{2}=0$, the system is uncontrollable.

(1.2) If $b_{1}=0$ and $b_{2} \neq 0$, the system is controllable if and only if

$$
\operatorname{det} \mathscr{C}_{1} \operatorname{det} \mathscr{C}_{2}>0 \text {. }
$$

(1.3) If $b_{1} \neq 0$, the system is controllable if and only if

$$
\operatorname{det} \mathscr{C}_{1} \operatorname{det} \mathscr{C}_{2}>0, \quad \operatorname{det}_{12}^{12} \mathscr{C}_{1} \operatorname{det}_{12}^{12} \mathscr{C}_{2}>0
$$

(2) If $r=1$, one has the following possibilities.

(2.1) If $b_{1}=0$, the system is uncontrollable.

(2.2) If $b_{1} \neq 0$, one distinguishes between the following corresponding four different reduced forms.

(a) If

$$
\begin{aligned}
A_{1}=\left(\begin{array}{ccc}
a_{1} & 0 & 0 \\
a_{2} & \lambda_{1} & 0 \\
a_{3} & 0 & \lambda_{1}
\end{array}\right), \quad A_{2}=\left(\begin{array}{ccc}
\bar{a}_{1} & 0 & 0 \\
\bar{a}_{2} & \lambda_{1} & 0 \\
\bar{a}_{3} & 0 & \lambda_{1}
\end{array}\right), \\
B=\left(\begin{array}{l}
b_{1} \\
b_{2} \\
b_{3}
\end{array}\right),
\end{aligned}
$$

then the system is uncontrollable.

(b) If

$$
\begin{aligned}
A_{1}=\left(\begin{array}{ccc}
a_{1} & 0 & 0 \\
a_{2} & \lambda_{1} & 0 \\
a_{3} & 1 & \lambda_{1}
\end{array}\right), \quad A_{2}=\left(\begin{array}{ccc}
\bar{a}_{1} & 0 & 0 \\
\bar{a}_{2} & \lambda_{1} & 0 \\
\bar{a}_{3} & 1 & \lambda_{1}
\end{array}\right), \\
B=\left(\begin{array}{l}
b_{1} \\
b_{2} \\
b_{3}
\end{array}\right),
\end{aligned}
$$

then the system is controllable if and only if

$$
\operatorname{det}_{12}^{12} \mathscr{C}_{1} \operatorname{det}_{12}^{12} \mathscr{C}_{2}>0 \text {; }
$$


moreover, $\operatorname{det} \mathscr{C}_{i}=\left(1 / b_{1}\right)\left(\operatorname{det}_{12}^{12} \mathscr{C}_{i}\right)^{2}, i=1,2$, so that $\operatorname{det} \mathscr{C}_{1}$ $\operatorname{det} \mathscr{C}_{2}>0$.

(c) If

$$
\begin{gathered}
A_{1}=\left(\begin{array}{ccc}
a_{1} & 0 & 0 \\
a_{2} & \lambda_{1} & 0 \\
a_{3} & 0 & \lambda_{2}
\end{array}\right), \quad A_{2}=\left(\begin{array}{ccc}
\bar{a}_{1} & 0 & 0 \\
\bar{a}_{2} & \lambda_{1} & 0 \\
\bar{a}_{3} & 0 & \lambda_{2}
\end{array}\right), \\
B=\left(\begin{array}{l}
b_{1} \\
b_{2} \\
b_{3}
\end{array}\right),
\end{gathered}
$$

$\lambda_{1}, \lambda_{2} \in \mathbb{R}$ with $\lambda_{1} \neq \lambda_{2}$, then the system is controllable if and only if

$$
\operatorname{det}_{12}^{12} \mathscr{C}_{1} \operatorname{det}_{12}^{12} \mathscr{C}_{2}>0, \quad \operatorname{det}_{12}^{13} \mathscr{C}_{1} \operatorname{det}_{12}^{13} \mathscr{C}_{2}>0 ;
$$

moreover, $\operatorname{det} \mathscr{C}_{i}=\left(\left(\lambda_{2}-\lambda_{1}\right) / b_{1}\right) \operatorname{det}_{12}^{12} \mathscr{C}_{i} \operatorname{det}_{12}^{13} \mathscr{C}_{i}, i=1,2$, so that $\operatorname{det} \mathscr{C}_{1} \operatorname{det} \mathscr{C}_{2}>0$.

(d) If

$$
\begin{gathered}
A_{1}=\left(\begin{array}{ccc}
a_{1} & 0 & 0 \\
a_{2} & \eta_{1} & \eta_{2} \\
a_{3} & -\eta_{2} & \eta_{1}
\end{array}\right), \quad A_{2}=\left(\begin{array}{ccc}
\bar{a}_{1} & 0 & 0 \\
\bar{a}_{2} & \eta_{1} & \eta_{2} \\
\bar{a}_{3} & -\eta_{2} & \eta_{1}
\end{array}\right), \\
B=\left(\begin{array}{l}
b_{1} \\
b_{2} \\
b_{3}
\end{array}\right),
\end{gathered}
$$

with $\eta_{2} \neq 0$, then the system is controllable.

Proof. We will go through the following different cases.

Case 1.1. It follows straightforward from Proposition 13.

Case 1.2. In this case we have

$$
\mathscr{C}_{1}=\left(\begin{array}{ccc}
0 & b_{2} & a_{1} b_{2} \\
b_{2} & 0 & a_{2} b_{2} \\
b_{3} & \lambda_{0} b_{3} & a_{3} b_{2}+\lambda_{0}^{2} b_{3}
\end{array}\right)
$$

and analogously for $\mathscr{C}_{2}$. On the other hand, for the triple of matrices (41), Condition (C2) of Theorem 4 can be rewritten as

$$
\begin{aligned}
\mu_{1} \mu_{2} & \\
& =\left(a_{1} v_{1}+a_{2} v_{2}+a_{3} v_{3}-\lambda v_{1}\right)\left(\bar{a}_{1} v_{1}+\bar{a}_{2} v_{2}+\bar{a}_{3} v_{3}-\lambda v_{1}\right) \\
& >0
\end{aligned}
$$

for any pair $\lambda \in \mathbb{R}, v \neq 0$ such that

$$
\lambda v_{2}-v_{1}=0, \quad \lambda v_{3}-\lambda_{0} v_{3}=0, \quad v_{2} b_{2}+v_{3} b_{3}=0
$$

and, as $b_{2} \neq 0$,

$$
v_{2}=-\frac{b_{3}}{b_{2}} v_{3}, \quad v_{1}=\lambda v_{2}=-\lambda \frac{b_{3}}{b_{2}} v_{3} .
$$

Hence, $v_{3} \neq 0$. Obviously, we can assume $v_{3}=1$, so that

$$
\begin{gathered}
\lambda=\lambda_{0}, \\
v=\left(-\lambda_{0} \frac{b_{3}}{b_{2}},-\frac{b_{3}}{b_{2}}, 1\right), \\
\mu_{1}=-\lambda_{0} \frac{b_{3}}{b_{2}} a_{1}-\frac{b_{3}}{b_{2}} a_{2}+a_{3}+\lambda_{0}^{2} \frac{b_{3}}{b_{2}}=-\frac{1}{b_{2}^{3}} \operatorname{det} \mathscr{C}_{1}, \\
\mu_{2}=-\frac{1}{b_{2}^{3}} \operatorname{det} \mathscr{C}_{2} ;
\end{gathered}
$$

therefore, Condition (C2) of Theorem 4 is equivalent to

$$
\operatorname{det} \mathscr{C}_{1} \operatorname{det} \mathscr{C}_{2}>0 \text {, }
$$

which obviously implies Condition (C1).

Case 1.3. First note that, as $b_{1} \neq 0$, we can assume $b_{1}=1$. Then,

$$
\mathscr{C}_{1}=\left(\begin{array}{ccc}
1 & a_{1}+b_{2} & a_{1}\left(a_{1}+b_{2}\right)+a_{2} \\
b_{2} & a_{2} & a_{2}\left(a_{1}+b_{2}\right) \\
b_{3} & a_{3}+\lambda_{0} b_{3} & a_{3}\left(a_{1}+b_{2}\right)+\lambda_{0}\left(a_{3}+\lambda_{0} b_{3}\right)
\end{array}\right) .
$$

In particular, $\operatorname{det}_{12}^{12} \mathscr{C}_{1}=a_{2}-b_{2}\left(a_{1}+b_{2}\right)$, so we can write $\operatorname{det} \mathscr{C}_{1}$ as $\operatorname{det} \mathscr{C}_{1}$

$$
\begin{aligned}
& =\operatorname{det}\left(\begin{array}{lll}
B & A_{1} B & A_{1}^{2} B-\left(a_{1}+b_{2}\right) A_{1} B
\end{array}\right) \\
& =\operatorname{det}\left(\begin{array}{ccc}
1 & a_{1}+b_{2} & \operatorname{det}_{12}^{12} \mathscr{C}_{1} \\
b_{2} & a_{2} & 0 \\
b_{3} & a_{3}+\lambda_{0} b_{3} & \lambda_{0}\left(a_{3}+\lambda_{0} b_{3}\right)-\lambda_{0} b_{3}\left(a_{1}+b_{2}\right)
\end{array}\right) \\
& =\left(b_{2} a_{3}-b_{3} a_{2}+\lambda_{0} a_{3}+\lambda_{0}^{2} b_{3}-\lambda_{0} b_{3} a_{1}\right) \operatorname{det}_{12}^{12} \mathscr{C}_{1}
\end{aligned}
$$

and analogously for $\mathscr{C}_{2}$. Now the pairs $\lambda \in \mathbb{R}, v \neq 0$ in Condition (C2) of Theorem 4 must verify

$$
\begin{gathered}
\lambda v_{2}-v_{1}=0, \quad \lambda v_{3}-\lambda_{0} v_{3}=0, \\
v_{1}+v_{2} b_{2}+v_{3} b_{3}=0 .
\end{gathered}
$$

For $v_{3}=0$, necessarily $v_{2} \neq 0$ (otherwise, $v_{1}=0$ by the first equation). We can assume $v_{2}=1$, so that

$$
\begin{gathered}
v=\left(-b_{2}, 1,0\right), \quad \lambda=-b_{2}, \\
\mu_{1}=-b_{2} a_{1}+a_{2}-b_{2}^{2}=\operatorname{det}_{12}^{12} \mathscr{C}_{1}, \quad \mu_{2}=\operatorname{det}_{12}^{12} \mathscr{C}_{2} .
\end{gathered}
$$

Thus, a necessary condition for the controllability of the system is

$$
\operatorname{det}_{12}^{12} \mathscr{C}_{1} \operatorname{det}_{12}^{12} \mathscr{C}_{2}>0 .
$$

Furthermore, we must consider the pairs $\lambda, v$ with $v_{3} \neq 0$. We can assume $v_{3}=1$, so that $\lambda=\lambda_{0}$ and

$$
v_{1}=\lambda_{0} v_{2}=-b_{2} v_{2}-b_{3} .
$$

Now we distinguish the following three situations, depending on the solutions of the last equation. 
(i) If $\lambda_{0}+b_{2}=0, b_{3} \neq 0$, none $v_{2}$ is a solution of (61), so that Condition (C2) of Theorem 4 is equivalent to

$$
\operatorname{det}_{12}^{12} \mathscr{C}_{1} \operatorname{det}_{12}^{12} \mathscr{C}_{2}>0
$$

but

$$
\begin{gathered}
\operatorname{det} \mathscr{C}_{1}=b_{3}\left(-a_{2}+b_{2}^{2}+b_{2} a_{1}\right) \operatorname{det}_{12}^{12} \mathscr{C}_{1}=-b_{3}\left(\operatorname{det}_{12}^{12} \mathscr{C}_{1}\right)^{2}, \\
\operatorname{det} \mathscr{C}_{2}=-b_{3}\left(\operatorname{det}_{12}^{12} \mathscr{C}_{2}\right)^{2} .
\end{gathered}
$$

Hence, Condition (C1) of Theorem 4 holds, and also $\operatorname{det} \mathscr{C}_{1} \operatorname{det} \mathscr{C}_{2}>0$.

(ii) If $\lambda_{0}+b_{2} \neq 0$, (61) has a unique solution:

$$
\begin{gathered}
v_{2}=-\frac{b_{3}}{\lambda_{0}+b_{2}}, \quad v_{1}=-\frac{\lambda_{0} b_{3}}{\lambda_{0}+b_{2}}, \\
\mu_{1}=-\frac{1}{\lambda_{0}+b_{2}}\left(a_{1} \lambda_{0} b_{3}+a_{2} b_{3}-a_{3}\left(\lambda_{0}+b_{2}\right)-\lambda_{0}^{2} b_{3}\right) \\
=\frac{1}{\lambda_{0}+b_{2}} \times \frac{\operatorname{det} \mathscr{C}_{1}}{\operatorname{det}_{12}^{12} \mathscr{C}_{1}}, \\
\mu_{2}=\frac{1}{\lambda_{0}+b_{2}} \times \frac{\operatorname{det}_{\mathscr{C}_{2}}}{\operatorname{det}_{12}^{12} \mathscr{C}_{2}} ;
\end{gathered}
$$

therefore, Condition (C2) of Theorem 4 is equivalent to

$$
\operatorname{det}_{12}^{12} \mathscr{C}_{1} \operatorname{det}_{12}^{12} \mathscr{C}_{2}>0, \quad \operatorname{det} \mathscr{C}_{1} \operatorname{det} \mathscr{C}_{2}>0
$$

which obviously implies Condition (C1).

(iii) Finally, if $\lambda_{0}+b_{2}=b_{3}=0$, we have infinitely many solutions of (61) and then

$$
\lambda=\lambda_{0}, \quad v=\left(\lambda_{0} v_{2}, v_{2}, 1\right), \quad v_{2} \in \mathbb{R} .
$$

Notice that

$$
\operatorname{det} \mathscr{C}_{1}=\operatorname{det} \mathscr{C}_{2}=0 \text {. }
$$

Hence, (43) does not hold. Let us see that conditions of Theorem 4 also fail. From Condition (C2), we have

$\mu_{1}=a_{1} \lambda_{0} v_{2}+a_{2} v_{2}+a_{3}-\lambda_{0}^{2} v_{2}=v_{2} \operatorname{det}_{12}^{12} \mathscr{C}_{1}+a_{3}$,

$$
\mu_{2}=v_{2} \operatorname{det}_{12}^{12} \mathscr{C}_{2}+\bar{a}_{3}
$$

and it follows that $\mu_{1} \mu_{2}>0$ holds for any $v_{2}$ if and only if

$$
a_{3} \bar{a}_{3}>0, \quad \frac{a_{3}}{\operatorname{det}_{12}^{12} \mathscr{C}_{1}}=\frac{\bar{a}_{3}}{\operatorname{det}_{12}^{12} \mathscr{C}_{2}},
$$

but then Condition (C1) fails, since

$$
\operatorname{rank}\left(B \quad A_{1} B \quad A_{1}^{2} B \text { e } A_{1} \text { e } A_{1}^{2} e\right)=2 .
$$

Indeed, the first two columns are linearly independent because (by hypothesis) $\operatorname{det}_{12}^{12} \mathscr{C}_{1} \neq 0$ and, as $\operatorname{det} \mathscr{C}_{1}=$ 0 , they span the third one. Finally, it is enough to verify that column $e$ is a linear combination of columns $B$ and $A_{1} B$, as direct computation shows

$$
\begin{aligned}
\operatorname{det}\left(\begin{array}{lll}
B & A_{1} B & e
\end{array}\right) & =\operatorname{det}\left(\begin{array}{ccc}
1 & a_{1}-\lambda_{0} & a_{1}-\bar{a}_{1} \\
-\lambda_{0} & a_{2} & a_{2}-\bar{a}_{2} \\
0 & a_{3} & a_{3}-\bar{a}_{3}
\end{array}\right) \\
& =-\operatorname{det}\left(\begin{array}{ccc}
1 & a_{1}-\lambda_{0} & \bar{a}_{1}-\lambda_{0} \\
-\lambda_{0} & a_{2} & \bar{a}_{2} \\
0 & a_{3} & \bar{a}_{3}
\end{array}\right) \\
& =a_{3} \operatorname{det}_{12}^{12} \mathscr{C}_{2}-\bar{a}_{3} \operatorname{det}_{12}^{12} \mathscr{C}_{1}=0 .
\end{aligned}
$$

Next, let us assume $r=1$. Then we have the following.

Case 2.1. This case follows from Proposition 13.

Case 2.2. (a) The proof proceeds in an analogous way to the proof of Case 1.2. We compute $\mu_{1}, \mu_{2}$ for any pair $\lambda \in \mathbb{R}, v \neq 0$ such that

$$
\begin{gathered}
\left(\lambda-\lambda_{1}\right) v_{2}=0, \quad\left(\lambda-\lambda_{1}\right) v_{3}=0, \\
v_{1} b_{1}+v_{2} b_{2}+v_{3} b_{3}=0 .
\end{gathered}
$$

Hence, if $\lambda \neq \lambda_{1}$, then $v_{2}=v_{3}=0$, and none $v \neq 0$ verifies the last equation. On the contrary, if $\lambda=\lambda_{1}$, by taking

$$
\begin{gathered}
v_{3}=-\left(a_{1}-\lambda_{1}\right) \frac{b_{2}}{b_{1}}+a_{2}, \quad v_{2}=\left(a_{1}-\lambda_{1}\right) \frac{b_{3}}{b_{1}}-a_{3}, \\
v_{1}=-\frac{b_{2}}{b_{1}} v_{2}-\frac{b_{3}}{b_{1}} v_{3}
\end{gathered}
$$

we have $\mu_{1}=0$. Therefore, Condition (C2) of Theorem 4 does not hold.

(b) For the triple of matrices of this case, we have

$$
\mathscr{C}_{1}=\left(\begin{array}{ccc}
b_{1} & a_{1} b_{1} & a_{1}^{2} b_{1} \\
b_{2} & a_{2} b_{1}+\lambda_{1} b_{2} & \left(a_{2} a_{1}+\lambda_{1} a_{2}\right) b_{1}+\lambda_{1}^{2} b_{2} \\
b_{3} & a_{3} b_{1}+b_{2}+\lambda_{1} b_{3} & \left(a_{3} a_{1}+a_{2}+\lambda_{1} a_{3}\right) b_{1}+2 \lambda_{1} b_{2}+\lambda_{1}^{2} b_{3}
\end{array}\right)
$$


and analogously for $\mathscr{C}_{2}$. Now, the pairs $\lambda \in \mathbb{R}, v \neq 0$ in Condition (C2) of Theorem 4 must verify

$$
\begin{gathered}
\left(\lambda-\lambda_{1}\right) v_{2}-v_{3}=0, \quad\left(\lambda-\lambda_{1}\right) v_{3}=0 \\
v_{1} b_{1}+v_{2} b_{2}+v_{3} b_{3}=0
\end{gathered}
$$

For $\lambda \neq \lambda_{1}$, the only solution is $v_{3}=v_{2}=v_{1}=0$. For $\lambda=\lambda_{1}$, there are infinitely many solutions:

$$
\begin{gathered}
v_{1}=-\frac{b_{2}}{b_{1}} v_{2}, \quad v_{2} \neq 0, \quad v_{3}=0, \\
\mu_{1}=\frac{v_{2}}{b_{1}}\left(a_{2} b_{1}-a_{1} b_{2}+\lambda_{1} b_{2}\right)=\frac{v_{2}}{b_{1}^{2}} \operatorname{det}_{12}^{12} \mathscr{C}_{1}
\end{gathered}
$$

and analogously for $\mu_{2}$. Therefore, Condition (C2) of Theorem 4 is equivalent to

$$
\operatorname{det}_{12}^{12} \mathscr{C}_{1} \operatorname{det}_{12}^{12} \mathscr{C}_{2}>0
$$

which implies Condition (C1) because

$$
\begin{aligned}
\operatorname{det} \mathscr{C}_{1} & =\operatorname{det}\left(\begin{array}{lll}
B & A_{1} B & A_{1}^{2} B
\end{array}\right) \\
& =\operatorname{det}\left(\begin{array}{lll}
B & A_{1} B & A_{1}^{2} B-\left(a_{1}+\lambda_{1}\right) A_{1} B+a_{1} \lambda_{1} B
\end{array}\right) \\
& =\operatorname{det}\left(\begin{array}{ccc}
b_{1} & a_{1} b_{1} & 0 \\
b_{2} & a_{2} b_{1}+\lambda_{1} b_{2} & 0 \\
b_{3} & a_{3} b_{1}+b_{2}+\lambda_{1} b_{3} & a_{2} b_{1}+\lambda_{1} b_{2}-a_{1} b_{2}
\end{array}\right) \\
& =\frac{1}{b_{1}}\left(\operatorname{det}_{12}^{12} \mathscr{C}_{1}\right)^{2} .
\end{aligned}
$$

(c) For the triple in this case, one sees that

$$
\mathscr{C}_{1}=\left(\begin{array}{ccc}
b_{1} & a_{1} b_{1} & a_{1}^{2} b_{1} \\
b_{2} & a_{2} b_{1}+\lambda_{1} b_{2} & a_{2} a_{1} b_{1}+\lambda_{1}\left(a_{2} b_{1}+\lambda_{1} b_{2}\right) \\
b_{3} & a_{3} b_{1}+\lambda_{2} b_{3} & a_{3} a_{1} b_{1}+\lambda_{2}\left(a_{3} b_{1}+\lambda_{2} b_{3}\right)
\end{array}\right)
$$

and analogously for $\mathscr{C}_{2}$. Now, the pairs $\lambda \in \mathbb{R}, v \neq 0$ in Condition (C2) of Theorem 4 must verify

$$
\begin{gathered}
\left(\lambda-\lambda_{1}\right) v_{2}=0, \quad\left(\lambda-\lambda_{2}\right) v_{3}=0, \\
v_{1} b_{1}+v_{2} b_{2}+v_{3} b_{3}=0 .
\end{gathered}
$$

If $v_{2}=v_{3}=0$, none $v \neq 0$ verifies the last equation. Hence, we can assume either $v_{2}=1$ or $v_{3}=1$, which yields, respectively,

$$
\begin{gathered}
v=\left(-\frac{b_{2}}{b_{1}}, 1,0\right), \quad \lambda=\lambda_{1}, \\
\mu_{1}=\frac{1}{b_{1}^{2}} \operatorname{det}_{12}^{12} \mathscr{C}_{1}, \quad \mu_{2}=\frac{1}{b_{1}^{2}} \operatorname{det}_{12}^{12} \mathscr{C}_{2}, \\
v=\left(-\frac{b_{3}}{b_{1}}, 0,1\right), \quad \lambda=\lambda_{2}, \\
\mu_{1}=\frac{1}{b_{1}^{2}} \operatorname{det}_{12}^{13} \mathscr{C}_{1}, \quad \mu_{2}=\frac{1}{b_{1}^{2}} \operatorname{det}_{12}^{13} \mathscr{C}_{2} .
\end{gathered}
$$

Therefore, Condition (C2) of Theorem 4 is equivalent to

$$
\operatorname{det}_{12}^{12} \mathscr{C}_{1} \operatorname{det}_{12}^{12} \mathscr{C}_{2}>0, \quad \operatorname{det}_{12}^{13} \mathscr{C}_{1} \operatorname{det}_{12}^{13} \mathscr{C}_{2}>0
$$

which implies Condition (C1) because

$$
\begin{aligned}
\operatorname{det} \mathscr{C}_{1} & =\operatorname{det}\left(\begin{array}{lll}
B & A_{1} B & A_{1}^{2} B-a_{1} A_{1} B
\end{array}\right) \\
& =\operatorname{det}\left(\begin{array}{ccc}
b_{1} & a_{1} b_{1} & 0 \\
b_{2} & a_{2} b_{1}+\lambda_{1} b_{2} & \frac{\lambda_{1}}{b_{1}} \operatorname{det}_{12}^{12} \mathscr{C}_{1} \\
b_{3} & a_{3} b_{1}+\lambda_{2} b_{3} & \frac{\lambda_{2}}{b_{1}} \operatorname{det}_{12}^{13} \mathscr{C}_{1}
\end{array}\right) \\
& =-\frac{\lambda_{1}-\lambda_{2}}{b_{1}} \operatorname{det}_{12}^{12} \mathscr{C}_{1} \operatorname{det}_{12}^{13} \mathscr{C}_{1} .
\end{aligned}
$$

(d) In this case, the pairs $\lambda \in \mathbb{R}, v \neq 0$ in Condition (C2) of Theorem 4 must verify

$$
\begin{gathered}
\left(\lambda-\eta_{1}\right) v_{2}+\eta_{2} v_{3}=0, \quad-\eta_{2} v_{2}+\left(\lambda-\eta_{1}\right) v_{3}=0 \\
v_{1} b_{1}+v_{2} b_{2}+v_{3} b_{3}=0
\end{gathered}
$$

Hence, $\left(\eta_{2}^{2}+\left(\lambda-\eta_{1}\right)^{2}\right) v_{3}=0$. As $\eta_{2} \neq 0$, the only solution is $v_{3}=0$, which implies $v_{2}=0$ and, in turn, $v_{1}=0$. Therefore, there is no pair $\lambda$, $\mu$ verifying Condition (C2) of Theorem 4. Condition ( $\mathrm{C} 1$ ) also holds, since the matrix consisting of the three first columns there has maximal rank. Indeed, a straightforward computation leads to

$$
\begin{aligned}
\operatorname{det} \mathscr{C}_{1} & =\operatorname{det}\left(\begin{array}{lll}
B & A_{1} B & A_{1}^{2} B
\end{array}\right) \\
& =\frac{1}{b_{1}^{2}} \operatorname{det}\left(\begin{array}{lll}
B & \left(A_{1} B-a_{1} B\right) b_{1} & A_{1}\left(A_{1} B-a_{1} B\right) b_{1}
\end{array}\right) \\
& =\frac{1}{b_{1}} \operatorname{det}\left(\begin{array}{cc}
\operatorname{det}_{12}^{12} \mathscr{C}_{1} & \eta_{1} \operatorname{det}_{12}^{12} \mathscr{C}_{1}+\eta_{2} \operatorname{det}_{12}^{13} \mathscr{C}_{1} \\
\operatorname{det}_{12}^{13} \mathscr{C}_{1} & -\eta_{2} \operatorname{det}_{12}^{12} \mathscr{C}_{1}+\eta_{1} \operatorname{det}_{12}^{13} \mathscr{C}_{1}
\end{array}\right) \neq 0
\end{aligned}
$$

because the second column is just the image of the first one by $\left(\begin{array}{cc}\eta_{1} & \eta_{2} \\ -\eta_{2} & \eta_{1}\end{array}\right)$, with $\eta_{2} \neq 0$.

Remark 20. We note that the use of the reduced forms in Propositions 18 and 19 leads to explicit and simple criteria (in comparison with [27]) for controllability of unobservable systems. These results, combined with the feedback induced unobservability stated in Proposition 17, provide an effective approach to the study of the controllability of the systems at hand. Of course, for piecewise linear systems, one can find in the literature a lot of examples of feedback design for several different purposes (for recent contributions, see [18-20]), but we have not found previous results on using feedbacks to discuss the controllability of a given system. In its current state, the approach presented here is valid for CBLCS. Possible extensions to general switched systems or to other interesting cases, such as systems with state delay, are subject of future research. 


\section{Example}

In this section, we will apply the results in Section 5 to a circuit analyzed in [4] (see Figure 1) which is an example of a 3D CBLCS that becomes unobservable for some values of the parameters. As we will see, the study of the controllability of this system will be an easy computation thanks to the use of Proposition 19.

Application of Kirchhoff laws yields

$$
\begin{gathered}
R_{2} C_{1} \dot{V}=-R_{2} i(V)-R_{2} I_{L}-V+V_{C_{2}} \\
L \dot{I}_{L}=V-I_{L} R_{1} \\
R_{2} C_{2} \dot{V}_{C_{2}}=V-V_{C_{2}},
\end{gathered}
$$

where

$$
i(V)= \begin{cases}\frac{V-V_{S} \operatorname{sgn}(V)}{R}, & \text { if }|V| \geq \frac{V_{S}}{2} \\ -\frac{V}{R}, & \text { if }|V|<\frac{V_{S}}{2}\end{cases}
$$

with $V_{S}$ being a constant saturation voltage (see [4] and the references therein for further details). Making the change of variables

$$
x=\left(x_{1}, x_{2}, x_{3}\right)=\frac{2}{V_{S}}\left(V, I_{L}, V_{C_{2}}\right),
$$

the system becomes

$$
\dot{x}=\left(\begin{array}{ccc}
-\frac{R+R_{2}}{R R_{2} C_{1}} & -\frac{1}{C_{1}} & \frac{1}{R_{2} C_{1}} \\
\frac{1}{L} & -\frac{R_{1}}{L} & 0 \\
\frac{1}{R_{2} C_{2}} & 0 & -\frac{1}{R_{2} C_{2}}
\end{array}\right) x+\left(\begin{array}{c}
\frac{2}{C_{1} R} \\
0 \\
0
\end{array}\right) \operatorname{sat}_{N}\left(x_{1}\right)
$$

when only positive values of $x_{1}$ are considered. Here, $\operatorname{sat}_{N}\left(x_{1}\right)$ denotes the normalized saturation function, defined by

$$
\operatorname{sat}_{N}(v)= \begin{cases}v, & \text { if }|v| \leq 1 \\ \operatorname{sgn}(v), & \text { if }|v| \geq 1\end{cases}
$$

We ask if the system is controllable by means of a control parameter $u$ acting as follows:

$$
\text { sat }\left(x_{1} ; u\right)= \begin{cases}x_{1}, & \text { if } x_{1} \leq u \\ u, & \text { if } x_{1} \geq u\end{cases}
$$

Now, introducing the new variable

$$
\bar{x}_{2}=-\frac{R+R_{2}}{R R_{2} C_{1}} x_{1}-\frac{1}{C_{1}} x_{2}+\frac{1}{R_{2} C_{1}} x_{3}
$$

yields

$$
\begin{aligned}
\left(\begin{array}{c}
\dot{x}_{1} \\
\dot{\bar{x}}_{2} \\
\dot{x}_{3}
\end{array}\right)= & \left(\begin{array}{ccc}
0 & 1 & 0 \\
\frac{-R R_{2}^{2} C_{2}-R_{1} R_{2} C_{2}\left(R+R_{2}\right)+R L}{R R_{2}^{2} C_{1} C_{2} L} & \frac{-L\left(R+R_{2}\right)-R R_{1} R_{2} C_{1}}{R R_{2} C_{1} L} & \frac{R_{1} R_{2} C_{2}-L}{R_{2}^{2} C_{1} C_{2} L} \\
\frac{1}{R_{2} C_{2}} & 0 & -\frac{1}{R_{2} C_{2}}
\end{array}\right)\left(\begin{array}{c}
x_{1} \\
\bar{x}_{2} \\
x_{3}
\end{array}\right) \\
& +\left(\begin{array}{c}
\frac{2}{C_{1} R} \\
-\frac{2\left(R+R_{2}\right)}{R^{2} R_{2} C_{1}^{2}} \\
0
\end{array}\right) \operatorname{sat}\left(x_{1} ; u\right) .
\end{aligned}
$$

Notice that the separating hyperplane is $x_{1}=u$; therefore, the shift $\bar{x}_{1}=x_{1}-u$ places it into $\bar{x}_{1}=0$. If, in addition, we write $\bar{x}_{3}=x_{3}$, then, in the new coordinates $\bar{x}=\left(\bar{x}_{1}, \bar{x}_{2}, \bar{x}_{3}\right)$, the above system takes the following form:

$$
\dot{\bar{x}}= \begin{cases}A_{1} \bar{x}+B u, & \text { if } \bar{x}_{1} \leq 0 \\ A_{2} \bar{x}+B u, & \text { if } \bar{x}_{1} \geq 0\end{cases}
$$


where

$$
\begin{gathered}
A_{1}=\left(\begin{array}{ccc}
\frac{2}{R C_{1}} & 1 & 0 \\
\frac{-R R_{2}^{2} C_{2}-R_{1} R_{2} C_{2}\left(R+R_{2}\right)+R L}{R R_{2}^{2} C_{1} C_{2} L}-\frac{2\left(R+R_{2}\right)}{R^{2} R_{2} C_{1}^{2}} & \frac{-L\left(R+R_{2}\right)-R R_{1} R_{2} C_{1}}{R R_{2} C_{1} L} & \frac{R_{1} R_{2} C_{2}-L}{R_{2}^{2} C_{1} C_{2} L} \\
\frac{1}{R_{2} C_{2}} & 0 & -\frac{1}{R_{2} C_{2}}
\end{array}\right), \\
A_{2}=\left(\begin{array}{ccc}
\frac{-R R_{2}^{2} C_{2}-R_{1} R_{2} C_{2}\left(R+R_{2}\right)+R L}{R R_{2}^{2} C_{1} C_{2} L} & \frac{-L\left(R+R_{2}\right)-R R_{1} R_{2} C_{1}}{R R_{2} C_{1} L} & \frac{R_{1} R_{2} C_{2}-L}{R_{2}^{2} C_{1} C_{2} L} \\
\frac{1}{R_{2} C_{2}} & 0 & -\frac{1}{R_{2} C_{2}}
\end{array}\right), \\
\quad\left(\begin{array}{cc}
\frac{2}{R C_{1}} \\
\frac{-R R_{2}^{2} C_{2}-R_{1} R_{2} C_{2}\left(R+R_{2}\right)+R L}{R R_{2}^{2} C_{1} C_{2} L}-\frac{2\left(R+R_{2}\right)}{R^{2} R_{2} C_{1}^{2}} \\
\frac{1}{R_{2} C_{2}}
\end{array}\right) .
\end{gathered}
$$

A simple computation shows that for $L=R_{1} R_{2} C_{2}$ the system is unobservable, having the following reduced form (see [15]):

$$
\begin{gathered}
A_{1}^{R}=\left(\begin{array}{ccc}
a_{1} & 1 & 0 \\
a_{2} & 0 & 0 \\
a_{3} & 0 & \lambda_{0}
\end{array}\right), \quad A_{2}^{R}=\left(\begin{array}{ccc}
\alpha_{1} & 1 & 0 \\
\alpha_{2} & 0 & 0 \\
\alpha_{3} & 0 & \lambda_{0}
\end{array}\right), \\
B^{R}=\left(\begin{array}{l}
b_{1} \\
b_{2} \\
b_{3}
\end{array}\right),
\end{gathered}
$$

where

$$
\begin{gathered}
\lambda_{0}=-\frac{1}{R_{2} C_{2}}, \quad a_{1}=\frac{2}{R C_{1}}-\frac{R C_{2}+R_{2} C_{2}+R C_{1}}{R R_{2} C_{1} C_{2}}, \\
a_{2}=\frac{R_{1}-R}{R R_{1} R_{2} C_{1} C_{2}}, \quad a_{3}=\frac{1}{R_{2} C_{2}}, \\
\alpha_{1}=-\frac{R C_{2}+R_{2} C_{2}+R C_{1}}{R R_{2} C_{1} C_{2}}, \quad \alpha_{2}=-\frac{R+R_{1}}{R R_{1} R_{2} C_{1} C_{2}}, \\
\alpha_{3}=\frac{1}{R_{2} C_{2}}, \quad b_{3}=\frac{1}{R C_{2}} .
\end{gathered}
$$

Thus, according to Case 1.3 in Proposition 19, the system will be controllable if and only if

$$
\operatorname{det} \mathscr{C}_{1} \operatorname{det} \mathscr{C}_{2}>0, \quad \operatorname{det}_{12}^{12} \mathscr{C}_{1} \operatorname{det}_{12}^{12} \mathscr{C}_{2}>0 .
$$

As in the proof there,

$$
\operatorname{det} \mathscr{C}_{1}=\left(b_{2} a_{3}-b_{3} a_{2}+\lambda_{0} a_{3} b_{1}+\lambda_{0}^{2} b_{3}-\lambda_{0} b_{3} a_{1}\right) \operatorname{det}_{12}^{12} \mathscr{C}_{1}
$$

and analogously for $\operatorname{det} \mathscr{C}_{2}$. In our case one obtains for the product

$$
\operatorname{det} \mathscr{C}_{1} \operatorname{det} \mathscr{C}_{2}=\frac{\left(R+R_{2}\right)^{2}}{R^{2} R_{2}^{4} C_{1}^{2} C_{2}^{3}} \operatorname{det}_{12}^{12} \mathscr{C}_{1} \operatorname{det}_{12}^{12} \mathscr{C}_{2} .
$$

Hence, we need to check only the second condition which, for the above reduced form, writes

$$
\begin{aligned}
\operatorname{det}_{12}^{12} \mathscr{C}_{1} \operatorname{det}_{12}^{12} \mathscr{C}_{2} & =\left(a_{2} b_{1}^{2}-a_{1} b_{1} b_{2}-b_{2}^{2}\right)\left(\alpha_{2} b_{1}^{2}-\alpha_{1} b_{1} b_{2}-b_{2}^{2}\right) \\
& >0
\end{aligned}
$$

but taking into account the values of the parameters in (97), it is equivalent to

$$
\begin{aligned}
\left(R_{1}-R\right) & {\left[2\left(R C_{2}+R_{2} C_{2}+R C_{1}\right) R_{1}-\left(R_{1}-R\right) R C_{1}\right] } \\
\times & {\left[-4\left(R+R_{1}\right) R_{1} R_{2} C_{2}\right.} \\
& +2\left(R_{1}-R\right)\left(R C_{2}+R_{2} C_{2}+R C_{1}\right) R_{1} \\
& \left.\quad-\left(R_{1}-R\right)^{2} R C_{1}\right]>0 .
\end{aligned}
$$

Clearly, the above inequality holds if $R_{1}<R$, but it does not hold if $R_{1}=R$, whereas for $R_{1}>R$, it holds or not depending on the other parameters. Thus, for $L=R_{1} R_{2} C_{2}$ we have the following. 
(i) If $R_{1}<R$, the system is controllable.

(ii) If $R_{1}=R$, the system is uncontrollable.

(iii) If $R_{1}>R$, then controllability requires additional conditions. For example, if

$R\left(R_{1}-R\right)>R_{2}\left(3 R+R_{1}\right) \quad$ or $\quad R_{1}-R>\frac{4 R_{1} R_{2} C_{2}}{R C_{1}}$ one sees at once that the system is controllable. On the other hand, necessary conditions for controllability are

$$
R_{1}\left(C_{1}+C_{2}\right)>R_{2} C_{2}, \quad \frac{2 C_{2}\left(R_{2}-R\right)}{R_{1}+R}<C_{1} .
$$

For $L \neq R_{1} R_{2} C_{2}$, the system is observable, and so to study the controllability we make use of Proposition 17 with $b_{1} \neq 0$. Hence (see Remark 16), the system is controllable if and only if the feedback system defined by $\left(A_{1}+B F, A_{2}+B F, B\right)$ is controllable, being

$$
\begin{aligned}
& F=\left(\begin{array}{lll}
0 & -\frac{1}{b_{1}} & 0
\end{array}\right)=\left(\begin{array}{lll}
0 & -\frac{1}{2} R C_{1} & 0
\end{array}\right), \\
& A_{1}+B F=\left(\begin{array}{c|cc}
\frac{2}{C_{1} R} & 0 & 0 \\
\hline \frac{-R R_{2}^{2} C_{2}-R_{1} R_{2} C_{2}\left(R+R_{2}\right)+R L}{R R_{2}^{2} C_{1} C_{2} L}-\frac{2\left(R+R_{2}\right)}{R^{2} R_{2} C_{1}^{2}} & \\
\frac{1}{R_{2} C_{2}} & \tilde{J}
\end{array}\right) \text {, } \\
& A_{2}+B F=\left(\begin{array}{c|cc}
0 & 0 & 0 \\
\frac{-R R_{2}^{2} C_{2}-R_{1} R_{2} C_{2}\left(R+R_{2}\right)+R L}{R R_{2}^{2} C_{1} C_{2} L} & \\
\frac{1}{R_{2} C_{2}} & \widetilde{J}
\end{array}\right), \\
& B=\left(\begin{array}{c}
\frac{2}{R C_{1}} \\
\frac{-R R_{2}^{2} C_{2}-R_{1} R_{2} C_{2}\left(R+R_{2}\right)+R L}{R R_{2}^{2} C_{1} C_{2} L}-\frac{2\left(R+R_{2}\right)}{R^{2} R_{2} C_{1}^{2}} \\
\frac{1}{R_{2} C_{2}}
\end{array}\right),
\end{aligned}
$$

where the block $\widetilde{J}$ in the matrices above is given by

$$
=\left(\begin{array}{cc}
\frac{-R_{1} R_{2}^{2} C_{2}+R R_{2}^{2} C_{2}+R R_{1} R_{2} C_{2}-R L}{2 R_{2}^{2} C_{2} L} & \frac{R_{1} R_{2} C_{2}-L}{R_{2}^{2} C_{1} C_{2} L} \\
-\frac{C_{1} R}{2 R_{2} C_{2}} & -\frac{1}{R_{2} C_{2}}
\end{array}\right) .
$$

It can be readily seen that this feedback system is unobservable with $r=1$, so that we can apply Proposition 19 again, now in the Case 2.2. In particular, it will be controllable if $\widetilde{J}$ has complex eigenvalues. For example,

(iv) if $L=R_{1} R_{2} C_{2} / 2$, the system is controllable if

$$
4 R R_{2}^{2}+4 R R_{1} R_{2}+R R_{1}^{2}<8 R_{1}^{2} R_{2}
$$

\section{Conclusions}

We obtain simple explicit criteria for the controllability of a continuous bimodal linear control system by means of the implicit ones in [27] and the reduced matrix forms in [15]. We cover fully dimensions $n=2$ and $n=3$, and partially higher ones. In particular, we prove that we can restrict ourselves to the nongeneric case of unobservable systems. Some interesting problems for further research could be the characterization of controllability for similar systems with multiple inputs and the extension of the present results to 


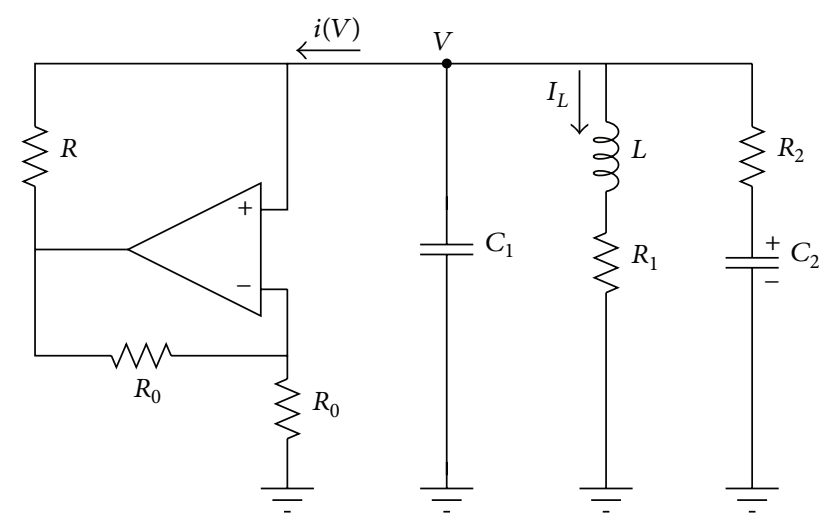

FIgURE 1: An occasional unobservable circuit.

systems with delays in the state variables, or even to general switched systems.

\section{Acknowledgments}

The second author was supported by the Spanish MINECOFEDER Grants MTM2009-06973 and MTM 2012-31714 and the Catalan CUR-DIUE Grant 2009SGR859. The other two authors were partially supported by the Project MTM 201123892.

\section{References}

[1] Z. Sun and S. S. Ge, Switched Linear Systems: Control and Design. Communications and Control Engineering, Springer, 2005.

[2] M. di Bernardo, C. J. Budd, A. R. Champneys, and P. Kowalczyk, Piecewise-Smooth Dynamical Systems, vol. 163 of Applied Mathematical Sciences, Springer, London, UK, 2008, Theory and applications.

[3] A. A. Andronov, A. A. Vitt, and S. E. Khaikin, Theory of Oscillators, Pergamon Press, Oxford, UK, 1966.

[4] V. Carmona, E. Freire, E. Ponce, and F. Torres, "On simplifying and classifying piecewise-linear systems," IEEE Transactions on Circuits and Systems, vol. 49, no. 5, pp. 609-620, 2002.

[5] E. Freire, E. Ponce, and J. Ros, "Limit cycle bifurcation from center in symmetric piecewise-linear systems," International Journal of Bifurcation and Chaos in Applied Sciences and Engineering, vol. 9, no. 5, pp. 895-907, 1999.

[6] V. Carmona, E. Freire, E. Ponce, J. Ros, and F. Torres, "Limit cycle bifurcation in 3D continuous piecewise linear systems with two zones. Application to Chua's circuit," International Journal of Bifurcation and Chaos in Applied Sciences and Engineering, vol. 15, no. 10, pp. 3153-3164, 2005.

[7] E. Freire, E. Ponce, and J. Ros, "The focus-center-limit cycle bifurcation in symmetric 3D piecewise linear systems," SIAM Journal on Applied Mathematics, vol. 65, no. 6, pp. 1933-1951, 2005.

[8] E. Freire, E. Ponce, and J. Ros, "A biparametric bifurcation in $3 \mathrm{D}$ continuous piecewise linear systems with two zones. Application to Chua's circuit," International Journal of Bifurcation and Chaos in Applied Sciences and Engineering, vol. 17, no. 2, pp. 445457, 2007.

[9] M. Komuro, R. Tokunaga, T. Matsumoto, L. O. Chua, and A. Hotta, "Global bifurcation analysis of the double scroll circuit,"
International Journal of Bifurcation and Chaos in Applied Sciences and Engineering, vol. 1, no. 1, pp. 139-182, 1991.

[10] J. Sotomayor and R. Garcia, "Structural stability of piecewiselinear vector fields," Journal of Differential Equations, vol. 192, no. 2, pp. 553-565, 2003.

[11] J. Llibre and J. Sotomayor, "Phase portraits of planar control systems," Nonlinear Analysis, vol. 27, no. 10, pp. 1177-1197, 1996.

[12] J. I. Imura and A. van der Schaft, "Characterization of wellposedness of piecewise-linear systems," IEEE Transactions on Automatic Control, vol. 45, no. 9, pp. 1600-1619, 2000.

[13] X. Puerta, "Feedback reduced and canonical forms for piecewise linear systems," in Proceedings of the 11th International Workshop on Variable Structure Systems (VSS '10), pp. 256-259, June 2010.

[14] J. Ferrer, M. D. Magret, J. R. Pacha, and M. Peña, "Planar bimodal piecewise linear systems. Bifurcation diagrams," Boletín de la Sociedad Española de Matemática Aplicada, no. 51, pp. 55-62, 2010.

[15] J. Ferrer, M. D. Magret, and M. Peña, "Bimodal piecewise linear dynamical systems. Reduced forms," International Journal of Bifurcation and Chaos in Applied Sciences and Engineering, vol. 20, no. 9, pp. 2795-2808, 2010.

[16] J. Xu and L. Xie, "Controllability and reachability of discretetime planar bimodal piecewise linear systems," in Proceedings of the American Control Conference, pp. 4387-4392, June 2006.

[17] J. Wen, L. Peng, and S. K. Nguang, "Receding horizon control for constrained jump bilinear systems," International Journal of Innovative Computing, Information and Control, vol. 8, no. 12, pp. 8501-8514, 2012.

[18] H. G. Zhang, G. T. Hui, and Y. C. Wang, "Stabilization of networked control systems with piecewise constant generalized sampled-data hold function," International Journal of Innovative Computing, Information and Control, vol. 9, no. 3, pp. 1159-1170, 2013.

[19] G. Zhai, T. Norisada, J. Imae, and T. Kobayashi, "An extension of generalized bilinear transformation for digital redesign," International Journal of Innovative Computing, Information and Control, vol. 8, no. 6, pp. 4071-4081, 2012.

[20] K. Liu, Y. Yao, D. Sun, and V. Balakrishnan, "Improved state feedback controller synthesis for piecewise-linear systems," International Journal of Innovative Computing, Information and Control, vol. 8, no. 10, pp. 6945-6957, 2012.

[21] M. K. Çamlibel, W. P. Heemels, and J. M. Schumacher, "Stability and controllability of planar bimodal linear complementarity systems," in Proceedings of the 42nd IEEE Conference on Decision and Control, pp. 1651-1656, December 2003.

[22] L. Wu, Z. Feng, and W. X. Zheng, "Exponential stability analysis for delayed neural networks with switching parameters: average dwell time approach," IEEE Transactions on Neural Networks, vol. 21, no. 9, pp. 1396-1407, 2010.

[23] L. Wu, X. Su, P. Shi, and J. Qiu, "A new approach to stability analysis and stabilization of discrete-time T-S fuzzy timevarying delay systems," IEEE Transactions on Systems, Man, and Cybernetics B, vol. 41, no. 1, pp. 273-286, 2011.

[24] M. K. Camlibel, W. P. Heemels, and J. M. Schumacher, "A full characterization of stabilizability of bimodal piecewise linear systems with scalar inputs," Automatica, vol. 44, no. 5, pp. 12611267, 2008.

[25] N. Cai, J. Xi, Y. Zhong, and H. Ma, "Controllability improvement for linear time-invariant dynamical multi-agent systems," International Journal of Innovative Computing, Information and Control, vol. 8, no. 5, pp. 3315-3328, 2012. 
[26] L. Wu, D. W. C. Ho, and C. W. Li, "Sliding mode control of switched hybrid systems with stochastic perturbation," Systems \& Control Letters, vol. 60, no. 8, pp. 531-539, 2011.

[27] M. K. Çamhbel, W. P. Heemels, and J. M. Schumacher, "On the controllability of bimodal piecewise linear systems," Lecture Notes in Computer Science, vol. 2993, pp. 250-264, 2004.

[28] R. E. Kalman, "On the general theory of control systems," in Proceedings of the 1st World Congress of the International Federation of Automatic Control, pp. 481-493, 1960.

[29] X. Su, P. Shi, L. Wu, and Y. D. Song, "A novel control design on discrete-time Takagi-Sugeno fuzzy systems with time-varying delays," IEEE Transactions of Fuzzy Systems, no. 99, 2012.

[30] X. Su, P. Shi, L. Wu, and Y. D. Song, "A novel approach to lter design for T-S fuzzy discrete-time systems with time-varying delay," IEEE Transactions on Fuzzy Systems, vol. 20, no. 6, pp. 1114-1129, 2012.

[31] L. Wu and J. Lam, "Sliding mode control of switched hybrid systems with time-varying delay," International Journal of Adaptive Control and Signal Processing, vol. 22, no. 10, pp. 909-931, 2008.

[32] L. Wu and W. X. Zheng, "Weighted $H_{\infty}$ model reduction for linear switched systems with time-varying delay," Automatica, vol. 45, no. 1, pp. 186-193, 2009.

[33] L. Wu, X. Su, and P. Shi, "Sliding mode control with bounded $L_{2}$ gain performance of Markovian jump singular time-delay systems," Automatica, vol. 48, no. 8, pp. 1929-1933, 2012. 


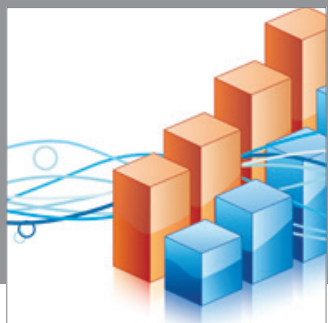

Advances in

Operations Research

mansans

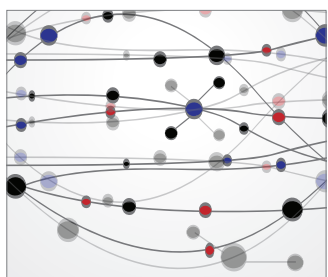

The Scientific World Journal
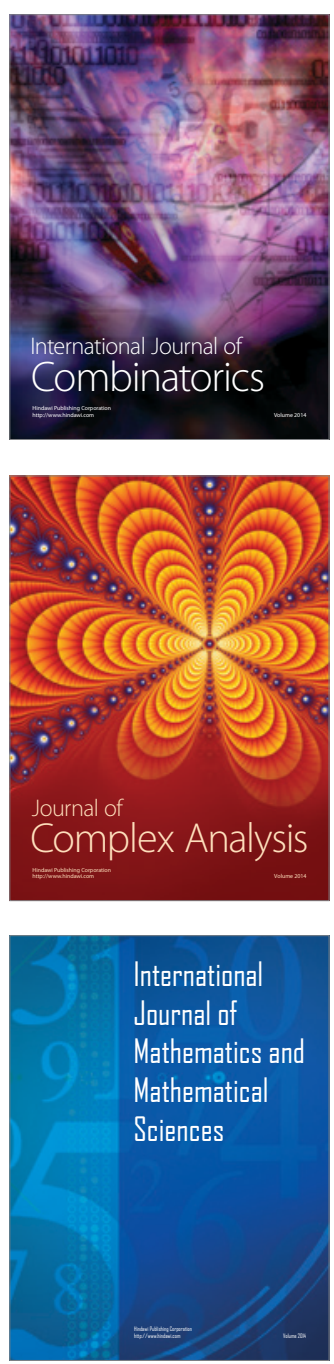
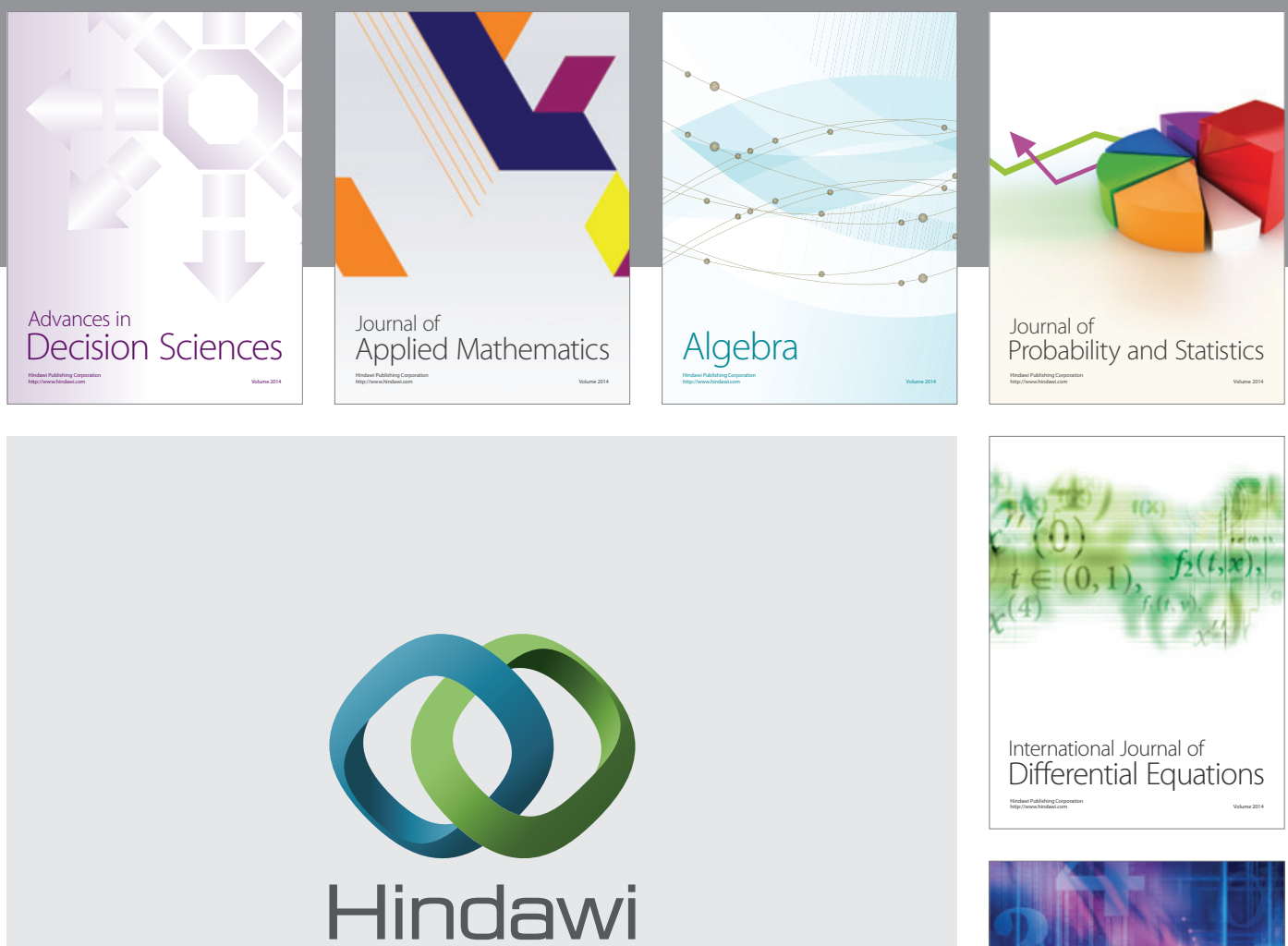

Submit your manuscripts at http://www.hindawi.com
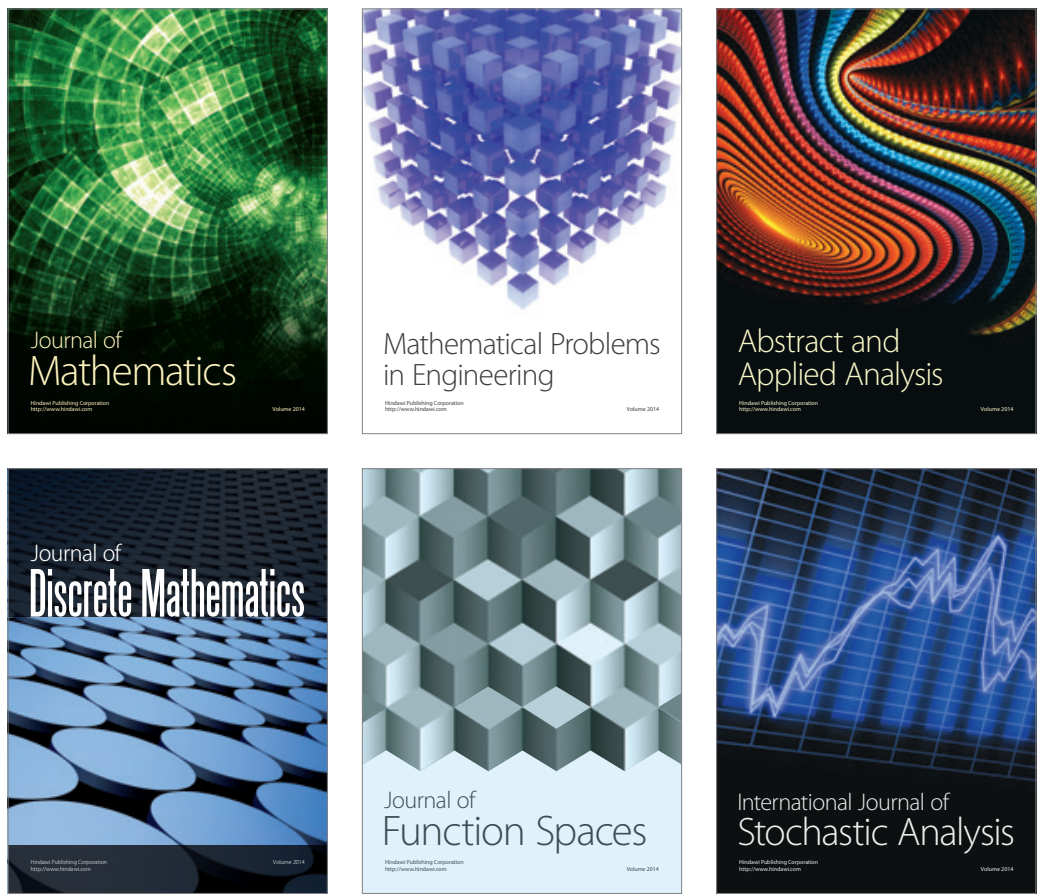

Journal of

Function Spaces

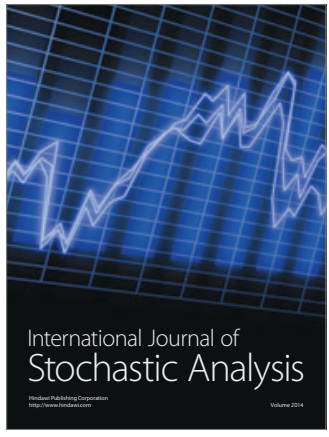

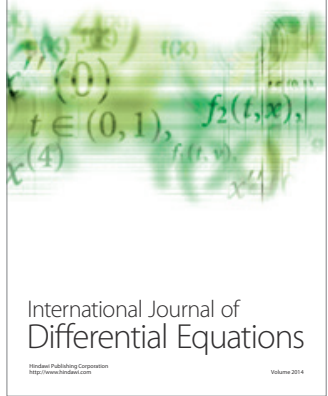
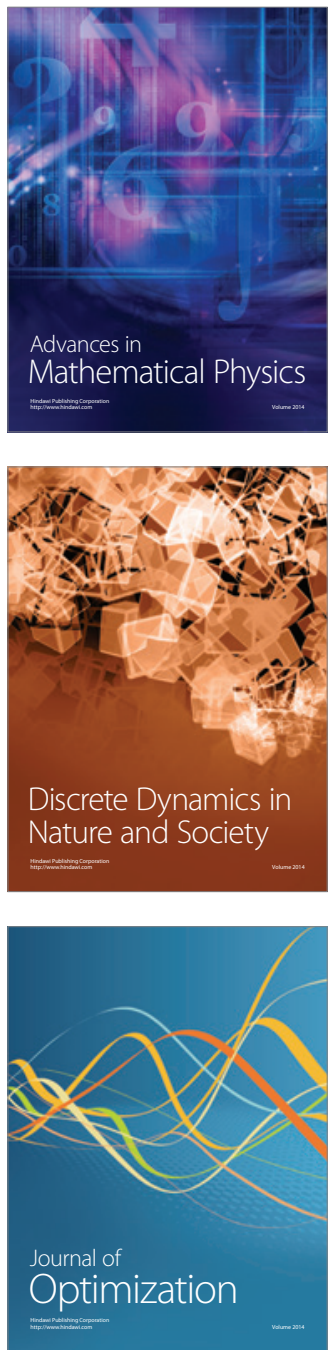